\title{
Treatment of Hematological Malignancies with Glycyrrhizic Acid
}

\author{
BRYAN J. HOSTETLER, OLGA N. UCHAKINA, HAO BAN and ROBERT J. MCKALLIP
}

Division of Basic Medical Sciences, Mercer University School of Medicine, Macon, GA, U.S.A.

\begin{abstract}
The current study examined the effectiveness of glycyrrhizic acid (GA) in reducing cell viability and inducing apoptosis in human chronic myeloid leukemia $(C M L)$ in vitro and a mouse lymphoma in vivo. Additionally, we assessed $G A$ as a candidate for combinational therapy in CML along with the current frontline treatment, imatinib (IM). Treatment of K562 CML cells with GA alone resulted in significant induction of apoptosis and loss of cell viability. GA was well tolerated by peripheral blood mononuclear cells (PBMCs) up to $2 \mathrm{mM}$ doses which were subsequently used in combination with IM. Co-treatment of CML with GA and IM greatly enhanced the levels of apoptosis in human CML. The effectiveness of GA was not limited to in vitro studies as treatment of EL-4 lymphoma-bearing mice with GA (50 or $500 \mathrm{mg} / \mathrm{kg} /$ day) led to significant dose-related decrease in tumor burden that correlated with a significant increase in the level of apoptotic tumors in vivo. The broad activity of GA against different tumor cell types, its tolerance by PBMCs and synergistic effects when combined with IM suggests that GA may be a viable candidate for combinational treatment strategies in CML and other hematological malignancies.
\end{abstract}

An estimated 170,000 people were expected to receive a diagnosis of lymphoma, leukemia, or myeloma in the United States in 2016 (1). The majority of these cancers will be treated with chemotherapeutics, radiation therapy, bone marrow transplant, or some combination thereof (2). Often the greatest hurdle in treatment is mathematics. It is estimated that tumor loads for hematological malignancies are between $1-3 \times 10^{12}$ cells depending on type and stage (3).

This article is freely accessible online.

Correspondence to: Robert J. McKallip, Mercer University School of Medicine, 1550 College St, Macon, GA, U.S.A. Tel: +1 4783012779, Fax: +1 4783015487, e-mail: mckallip_r@mercer.edu

Key Words: Glycyrrhizic acid, hyaluronic acid, chronic myelogenous leukemia, apoptosis.
Against trillions of malignant cells even the most specific therapies leave millions of untouched cells. Dosage of chemotherapeutics effective against such high tumor loads must be balanced against systemic toxicity which often leads to adverse results on both fronts. Inadequate treatment of the neoplasm along with life-threatening side-effects have augmented the need to develop targeted treatments and combination therapies.

A major advancement in the treatment of hematological malignancies was the development of imatinib as a targeted chronic myeloid leukemia therapy (CML). Its introduction as the standard CML treatment resulted in low cytotoxic effects on normal tissue and patient survival rates that closely mirrored the general population (4). Advances in targeted therapy have also been made in a subtype of acute promyelocytic leukemia, and hairy cell leukemia (5). Unfortunately, even with these advances the response to both broad and targeted therapies are often transient and treatment cessation or development of resistance leads to relapse. Highly effective targeted therapies such as imatinib in CML fail to eradicate the disease and require lifelong treatment. Cessation of therapy often results in relapse within 3 months and an estimated $20-30 \%$ of CML patients will develop resistance and disease progression (6-8).

Combination therapy is becoming the standard-of-care in the majority of cancer treatments as it aims increase efficacy and decrease toxicity. The challenge of targeting high tumor loads becomes feasible by utilizing multiple vectors near their max therapeutic dose. Several advances using combinational therapies have been reported and show great promise in the treatment of hematological malignancies (9). For example, combination therapy with hypomethylating agents, histone deacetylase inhibitors, along with other chemotherapeutic agents has shown promise over monotherapies in the treatment of myelodysplastic syndromes (10). This approach is also being explored in the treatment of drug-resistant CML. For example, use of inhibitors targeting JAK2 and HSP90 have shown promise in treating imatinib-resistant tumor cells (11). While other studies examining the development of resistance 
demonstrated that use of the combination of doxorubicin and curcumin as a single nanoparticle suppressed the development of multidrug resistance in a CML cell line (12).

GA is a triterpene glycoside complex isolated from licorice that was previously reported to possess antiinflammatory and anti-tumor properties (13). GA has been shown to be effective against a number of cancers including, but not limited to colon cancer, glioblastoma, lung cancer, leukemia, and melanoma (6, 14-18). Furthermore, GA was shown to clinically reduce the incidence of liver carcinogenesis in patients with hepatitis C (19). In addition, glycrrhetic acid, an aglycon of GA, has been demonstrated to have apoptotic effects on human hepatoma, promyelocytic leukemia, and stomach cancer cells in vitro (8). The mechanisms of GA activity are still under investigation, but its effectiveness against a wide variety of tumor cell types and previous reports of toleration by normal human leukocytes make it an attractive agent for combinational therapy (8). In the current study, we explored the effectiveness of GA in the treatment of both CML in vitro and lymphoma in vivo, its toleration by PBMCs ex vivo, and as co-therapy agent along with IM in the treatment of CML.

\section{Materials and Methods}

Reagents. Glycrrhizic acid (GA) was obtained from Spectrum Chemical (New Brunswick, NJ, USA). Imatinib was provided by Novartis (Basel, Switzerland). Antibodies for PARP and $\beta$-actin were obtained from Cell Signaling Technology (Beverly, MA, USA).

Cell lines. K562 and EL-4 cell lines were maintained in RPMI 1640 (Gibco Laboratories, Grand Island, NY, USA) and DMEM (Corning, Corning, NY, USA) respectively, supplemented with $10 \%$ fetal calf serum, $10 \mathrm{mM}$ HEPES, 1\% streptomycin/penicillin. EL-4 was passaged in vivo one time before use.

Measurement of the effect of imatinib and glycrrhizic acid on cell viability in vitro. K562 cells $\left(0.5 \times 10^{6}\right.$ cells/well) were cultured for $48 \mathrm{~h}$ in presence of GA $(1.0-4.0 \mathrm{mM})$, IM $(0.4 \mu \mathrm{M})$, or a combination $(2.0 \mathrm{mM} \mathrm{GA}, 0.4 \mu \mathrm{M}$ IM). Cells were harvested washed twice in PBS and viable cell count was determined by trypan blue dye exclusion assay.

Measurement of effect of imatinib and glycrrhizic acid on apoptosis in vitro. K562 cells $\left(0.5 \times 10^{6}\right.$ cells/well $)$ were cultured for $48 \mathrm{~h}$ in the presence of GA (1.0-4.0 mM), IM $(0.4 \mu \mathrm{M})$, or a combination (2.0 mM GA, $0.4 \mu \mathrm{M}$ IM). Cells were harvested, washed twice with PBS and prepared for apoptotic analysis using Annexin V/PI, DCF, TUNEL, or $\mathrm{DiOC}_{6}$ assays. To detect apoptosis by Annexin V/PI cell cultures were stained with FITC-labeled Annexin V and PI using the TAACS Annexin V-FITC kit by Trevigen (Gaithersburg, MD, USA). TUNEL was carried out using the Dead End Fluorometric TUNEL system by Promega (Madison, WI, USA).

Analysis of mitochondrial membrane potential $(\Delta \psi m)$. K562 cells $\left(0.5 \times 10^{6}\right.$ cells/well $)$ were cultured for $48 \mathrm{~h}$ in the presence of GA (1.0-4.0 mM), IM $(0.4 \mu \mathrm{M})$, or a combination (2.0 mM GA, $0.4 \mu \mathrm{M}$

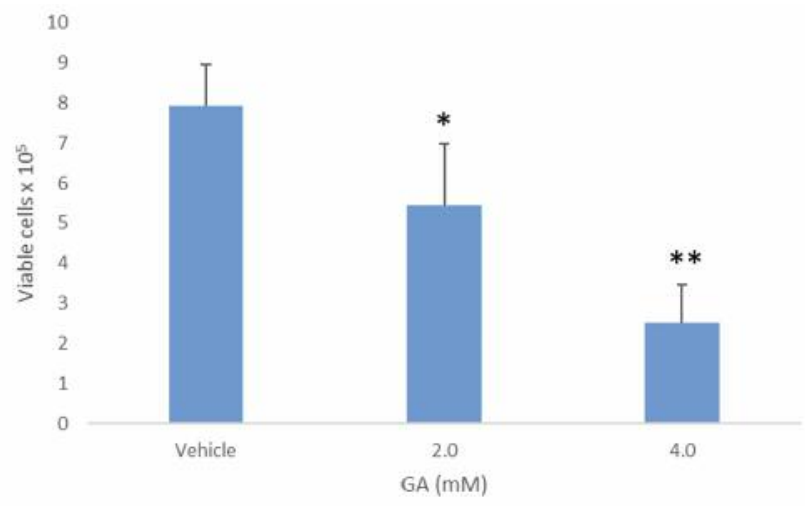

Figure 1. Glycrrhizic acid treatment leads to reduced cell viability in K562 CML cells in vitro. K562 cells were cultured for $48 \mathrm{~h}$ in the presence of vehicle control or GA (2.0 or $4.0 \mathrm{mM})$. Viable cell number was determined by trypan blue dye exclusion. The graph depicts the average $\pm S D$. of triplicate cultures. Asterisk denotes statistical significance $p \leq 0.05$. Double asterisk denotes statistical significance $p \leq 0.01$.

IM). To assess loss of mitochondrial membrane potential, 3,3'dihexylcarbocyanine iodide $\left(\mathrm{DiOC}_{6}\right.$, Molecular Probes, Inc., Eugene, OR, USA) was added to the cells for a final concentration of $40 \mathrm{nM}, 15 \mathrm{~min}$ prior to the end of the incubation. The cells were then harvested and analyzed using a flow cytometer (20).

ROS generation. K562 cells $\left(0.5 \times 10^{6}\right)$ were labeled for $1 \mathrm{~h}$ with $5 \mu \mathrm{M}$ carboxy-H2DCFDA (Molecular Probes). Next, excess carboxyH2DCFDA was removed by washing the cells and then suspending them in RPMI. The labeled cells were then exposed to vehicle control (DMSO) or various concentrations of GA (2 or $4 \mathrm{mM}$ ) for $48 \mathrm{~h}$ and the levels of ROS were determined by flow cytometric analysis using a FACSAria II flow cytometer (BD Biosciences, San Jose, CA, USA). In all experiments, $10^{4}$ viable cells were analyzed using forward/sidescatter gating.

Western blot. Proteins were extracted from K562 cells using RIPA buffer (Pierce, Rockford, IL, USA) supplemented with $1 \%$ protease inhibitor cocktail (Sigma-Aldrich, St. Louis, MO, USA), 1\% PMSF, and $1 \% \mathrm{NaF}$. Cell lysates were resolved in $10 \%$ polyacrylamide SDS gels and proteins were transferred to polyvinylidene fluoride membrane. The membrane was blocked with 5\% non-fat dry milk (Bio-Rad, Hercules, CA, USA) for $1 \mathrm{~h}$ at room temperature. Primary antibodies against PARP $(1: 1,000)$ and $\beta$-actin $(1: 2,000)$ (Cell Signaling Technology, Danvers, MA, USA) were used to probe the membrane in TBS overnight at $4^{\circ} \mathrm{C}$. TBS with $0.1 \%$ Tween 20 was used to wash the membrane 3 times before probing with HRP-conjugated secondary antibody for $1 \mathrm{~h}$ at room temperature. The proteins of interest were illuminated through incubation with ECL Western Blotting Substrate (Pierce) and exposed to X-ray film (Thermo, Waltham, MA, USA).

Measurement of glycrrhizic acid on cell viability and apoptosis in vivo. 12-14-week-old female mice, C57BL/6J (Jackson Laboratories, Bar Harbor, ME, USA) were injected i.p. with $200 \mu \mathrm{l}$ of EL-4 cells $\left(5.0 \times 10^{6}\right.$ cells $\left./ \mathrm{ml}\right)$ for a total of $1 \times 10^{6}$ cells. Starting 
A

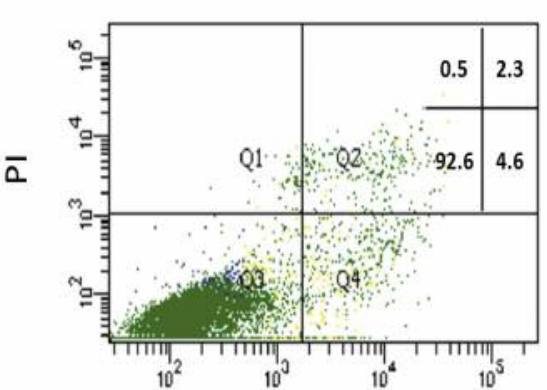

$2.0 \mathrm{mM} \mathrm{GA}$

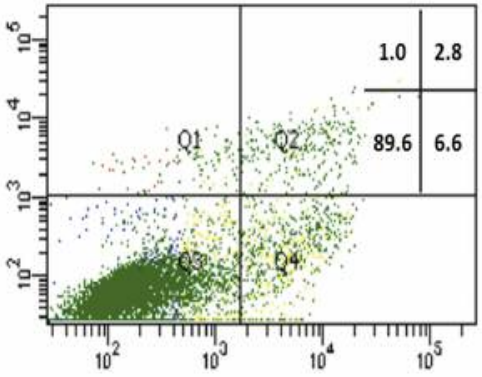

Annexin V
4.0 mM GA

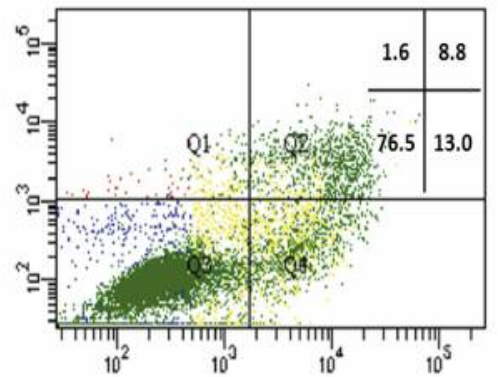

B

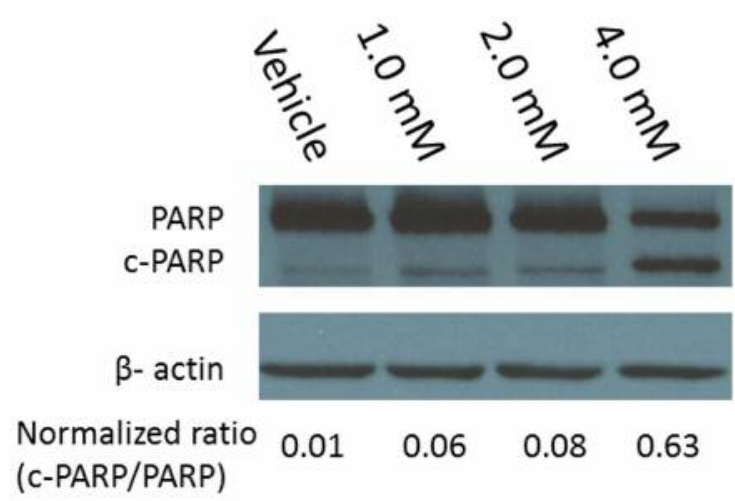

Figure 2. Glycrrhizic acid (GA) induces apoptosis in K562 CML cells in vitro. The effect of GA treatment on the induction of apoptosis in K562 cells was determined by treating the cells with various concentrations of GA (1.0, 2.0, or $4.0 \mathrm{mM})$ or vehicle control for $48 \mathrm{~h}$ and quantifying apoptosis using by Annexin V/PI staining (A), and PARP cleavage (B).

on day six, the mice were injected i.p. with PBS, $50 \mathrm{mg} / \mathrm{kg}$, or 500 $\mathrm{mg} / \mathrm{kg}$ GA once daily for four days. On day 10 the mice were sacrificed and the EL-4 cells were recovered by flushing the peritoneal cavity with $5 \mathrm{ml}$ of PBS. After recovery, cell viability was determined by trypan blue dye exclusion using a TC20 Automated Cell Counter (Bio-Rad, Hercules, CA, USA) at a size gating of $8-17 \mu \mathrm{m}$. The cells isolated from the peritoneal cavity were then stained with antibodies directed against Thy1.1 which is expressed by EL-4 tumor cells, and assayed for apoptosis using the TUNEL assay.

Statistical analysis. Results are presented as mean \pm standard deviation. Statistical analysis was performed using the Data Analysis ToolPak Excel Add-in. Student's two-tailed $t$-test or ANOVA were used where appropriate. Asterisks denote a p-value less than 0.05 and double asterisks denote a $p$-value less than 0.01 .

\section{Results}

Glycrrhizic acid treatment leads to reduced cell viability in K562 CML cells in vitro. Experiments were conducted to determine whether GA treatment affected K562 CML cell viability. K562 cells were treated under various concentrations of GA $(0,1.0,2.0$, or $4.0 \mathrm{mM})$ for $48 \mathrm{~h}$ (Figure 1). Cell viability was determined by trypan blue dye exclusion. The results demonstrated that K562 cell viability was significantly reduced at concentration of GA $>2.0 \mathrm{mM}$. More specifically, cell viability was significantly reduced from $7.9 \times 10^{5}$ to $5.4 \times 10^{5}$ and $2.5 \times 10^{5}$ following treatment with $2 \mathrm{mM}$ and $4 \mathrm{mM}$ GA, respectively, suggesting that CML cells are sensitive to GA treatment.

Glycrrhizic acid induces apoptosis in K562 CML cells in vitro. To determine whether GA led to reduced cell viability through induction of apoptosis, K562 cells were treated with various concentrations of GA $(1.0,2.0,4.0 \mathrm{mM})$ for $48 \mathrm{~h}$. The percentage of cells undergoing apoptosis was determined by Annexin V/PI staining (Figure 2A) and confirmed by examining PARP cleavage (Figure 2B). Annexin V/PI staining demonstrated a dose-related increase in the percentage of apoptotic tumor cells following GA treatment. There was a moderate increase in the early apoptotic cell population from the vehicle $(4.6 \%)$ to $2 \mathrm{mM}$ GA $(6.6 \%)$ and substantial shift at $4 \mathrm{mM}$ GA (13\%). 


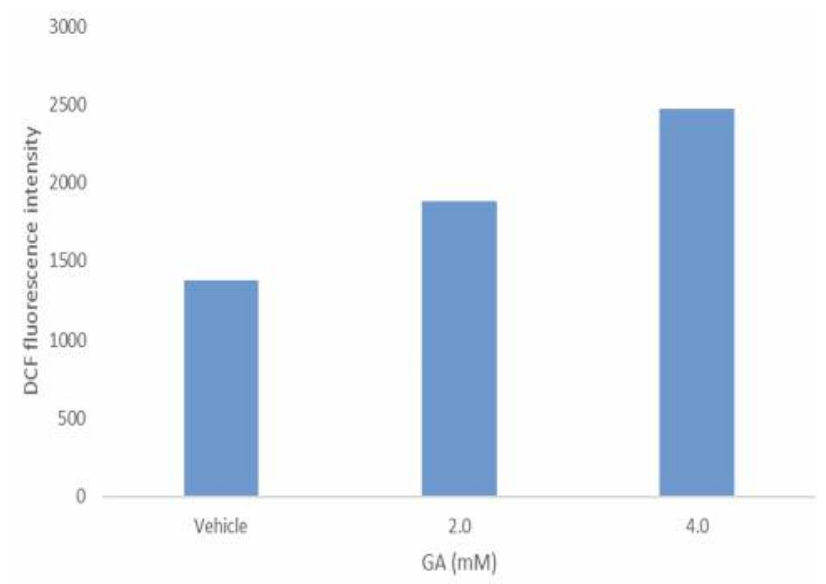

Figure 3. Exposure of K562 CML leads to increased levels of ROS. The effect of GA treatment on intracellular levels of ROS was determined by treating DCFDA-labeled K562 cells with various concentrations of GA (2.0 or $4.0 \mathrm{mM})$ or vehicle control for $48 \mathrm{~h}$ and determining ROS levels by flow cytometric analysis. The graph depicts the DCFDA fluorescence intensity. Data depicted are representative results from experiments that were conducted at least three times.

Analysis of PARP cleavage revealed that a dose of $4 \mathrm{mM}$ resulted in a large shift in the normalized ratio of cPARP/PARP from 0.01 in the vehicle to 0.63 in the GAtreated groups (Figure $2 \mathrm{~B}$ ). These data demonstrate that treatment of K562 cells with GA results in the induction of apoptosis.

Exposure of K562 CML leads to increased levels of ROS. Previous reports suggest that GA may kill tumor cells through ROS generation (7). To explore this mechanism K562 cells were treated with GA $(2.0,4.0 \mathrm{mM})$ for $48 \mathrm{~h}$ and then examined for changes intracellular levels of ROS by flow cytometric analysis. The results showed that ROS generation was greatly increased following GA treatment (Figure 3). However, the addition of the antioxidant $\mathrm{N}$ acetylcysteine (NAC) did not reduce cellular ROS (data not shown). Together, these results suggest that treatment with GA leads to oxidative stress that may not be related to GAinduced cell death.

EL-4 lymphoma are susceptible to GA in vivo. The ability of GA to induce apoptosis in lymphoma was examined in vivo. C57BL/6 mice were injected i.p. with the syngeneic tumor EL-4 cells $\left(1 \times 10^{6}\right.$ in $200 \mu \mathrm{l}$ PBS $)$. On days 6-9 the mice received daily injections of the vehicle or GA $(50$, or 500 $\mathrm{mg} / \mathrm{kg}$ i.p.) in groups of four. The tumor cells were recovered on day 10 and tumor burden and level of apoptotic tumor cells were determined. The results demonstrated that treatment with $500 \mathrm{mg} / \mathrm{kg}$ GA resulted in a significant reduction in tumor burden when compared to the vehicle-treated mice reducing the total tumor cell number by approximately $63 \%$ from $18.7 \times 10^{6} / \mathrm{ml}$ in the vehicle-treated mice to $6.9 \times 10^{6} / \mathrm{ml}$ in GAtreated mice (Figure 4A). The recovered cells were then stained with $\mathrm{mAb}$ directed against the EL-4 specific antibody Thy 1.1 and the level of apoptotic tumor cells was determined by TUNEL assay (Figure 4B, C). GA treatment resulted in dose-related increase in TUNEL positive EL-4 tumor cells. More specifically, treatment with $50 \mathrm{mg} / \mathrm{kg}$ GA led to a moderate increase in apoptotic tumor cells from $17.3 \pm 0.97 \%$ in the vehicle to $23.1 \pm 6.87 \%$ while treatment with $500 \mathrm{mg} / \mathrm{kg}$ led to a significant increase to $35.5 \pm 8.35 \%$.

Co-treatment of K562 with GA and IM enhances levels of apoptosis in vitro. We next examined whether a $2.0 \mathrm{mM}$ dose of GA was a candidate for combination with imatinib as the frontline mono-therapy treatment in CML. K562 cell cultures were treated with $2.0 \mathrm{mM}$ GA, $0.4 \mu \mathrm{M}$ IM, or a combination of the two for $48 \mathrm{~h}$ and analyzed for apoptosis using the TUNEL assay. Combining IM with GA treatment resulted in greatly increased DNA fragmentation (19.6\%) when compared to GA $(8.1 \%)$ or IM $(6.7 \%)$ alone (Figure $5 \mathrm{~A})$. Cultures were also assayed for loss of mitochondrial membrane potential (Figure 5B). The data was consistent with the TUNEL assay showing a membrane potential loss in the combination treatment of $49.6 \%$ compared to $23.5 \%$ in $\mathrm{GA}$, or $34.1 \%$ in IM alone (Figure $5 \mathrm{~B}$ ).

Low doses of GA are tolerated by human PBMC's. After establishing the potent therapeutic effects of GA on K562 in vitro and EL-4 in vivo we explored whether our current dosages were tolerated by non-cancerous cells. PBMCs were isolated from blood samples taken from healthy donors and exposed to GA $(0.25-4.0 \mathrm{mM})$ for $24 \mathrm{~h}$. The PBMCs were assayed for cell viability and induction of apoptosis. GA had little effect on cell viability of PBMCs from both donors including at the highest doses of 2.0 and $4.0 \mathrm{mM}$ (Figure 6). The PBMCs were also assayed for apoptosis by Annexin V/PI staining. Contrary to cell viability, both early (Annexin $\mathrm{V}^{+} \mathrm{PI}^{-}$) and late apoptosis/necrosis (Annexin $\mathrm{V}^{+} \mathrm{PI}^{+}$) was greatly increased in cells treated with $4.0 \mathrm{mM}$ GA while all doses $2.0 \mathrm{mM}$ and below appeared to have little effect.

\section{Discussion}

In the current study, we examined the effects of GA on hematological malignancies including a human CML and mouse lymphoma. Treatment with GA led to induction of apoptosis in human CML in vitro and similarly to reduction in tumor burden through induction of apoptosis in EL-4 tumor-bearing mice. In contrast, human PBMC seemed to be relatively resistant to the effects of GA. Furthermore, we demonstrated that GA may be efficacious when used as a cotreatment with IM for treating CML. 
A

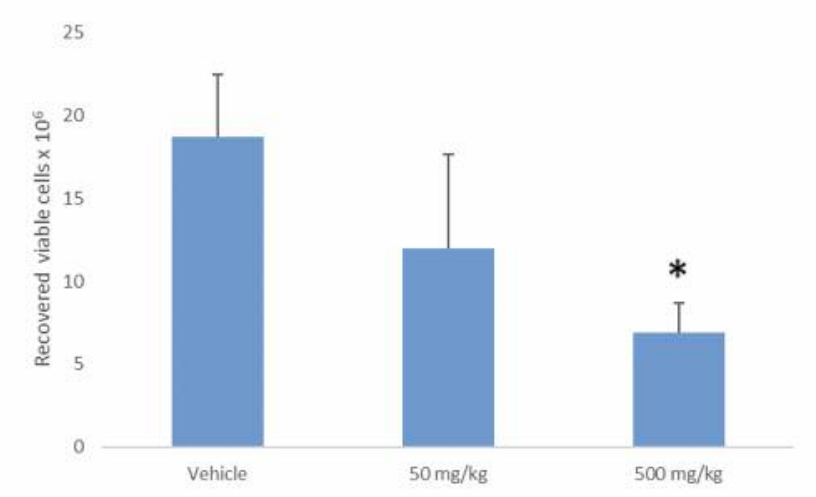

B

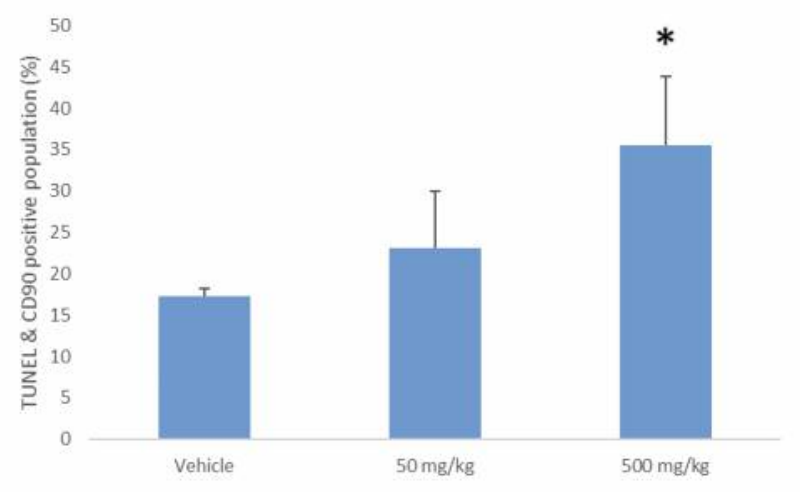

C

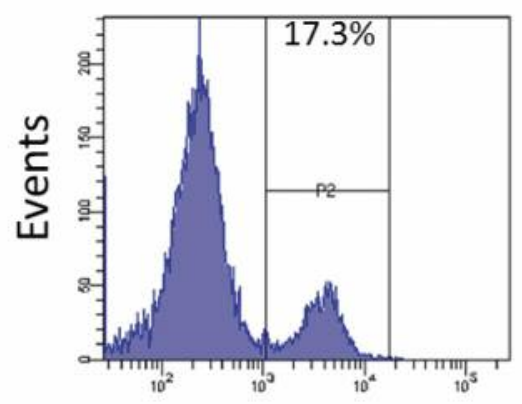

$50 \mathrm{mg} / \mathrm{kg}$

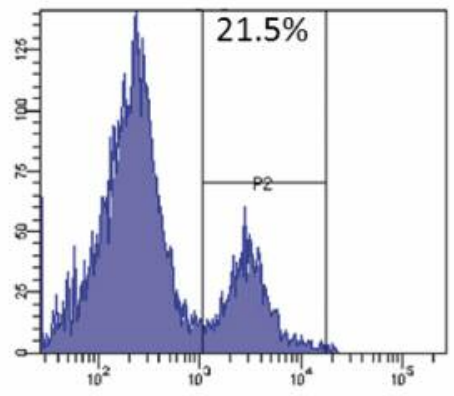

TUNEL positive

Figure 4. EL-4 lymphoma are susceptible to glycrrhizic acid in vivo. The effect of GA treatment on EL-4 tumor growth was determined. Mice were injected i.p. with EL-4 tumor cells. The tumor-bearing mice received GA (vehicle, 50 or $500 \mathrm{mg} / \mathrm{kg}$ ) treatment on days 6 through 9 . On day 10 the tumor cells were harvested and the viable cell count was determined by trypan blue dye exclusion $(A)$. The effect of GA treatment was determined by TUNEL assay $(B)$. Asterisk represents a statistically significant difference $(p<0.05)$ when compared to vehicle treated mice. A representative TUNEL staining is depicted in panel $C$.

Licorice root and its extracts have been used therapeutically for centuries as a treatment for ulcers, inflammation, viral infections, arthritis, as well as cancer (21). GA and its derivatives are reported to be the active compounds in licorice root (22). There is a number of reports demonstrating anticancer properties of GA. Specifically, GA has been shown to be effective at inhibiting the growth of colon cancer, glioblastoma, lung cancer, melanoma, and ovarian cancer cell lines $(6,14-18)$. In addition, glycyrrhetic acid, a derivative of GA, is active against human hepatoma, promyelotic leukemia, and stomach cancer cell lines in vitro (8). Glycyrrhetinic acid, another metabolite of GA, was found to reduce the proliferation rate of prostate cancer cells (23). Clinically, treatment of patients with chronic hepatitis with GA led to significantly reduced rates of hepatocarcinogenesis
(19). Little has been reported regarding the effectiveness of GA in treating hematological malignancies such as CML. However, there are a limited number of reports of GA being effective against leukemias. Chueh et al. reported that exposure of the mouse leukemia cell line WEHI-3 led to cell cycle arrest and the induction of apoptosis (24). In addition, $\mathrm{He}$ et al. reported that GA treatment led to reduced proliferation of the human leukemia cell line TF-1 and that GA treatment inhibited TF-1 growth in vivo (7). However, prior to our study information regarding the effectiveness of GA on CML had not reported. Our findings that GA effectively reduces CML proliferation in vitro and reduces leukemia tumor load in vivo provide additional support for the possible use of GA in the treatment of hematological malignancies. 

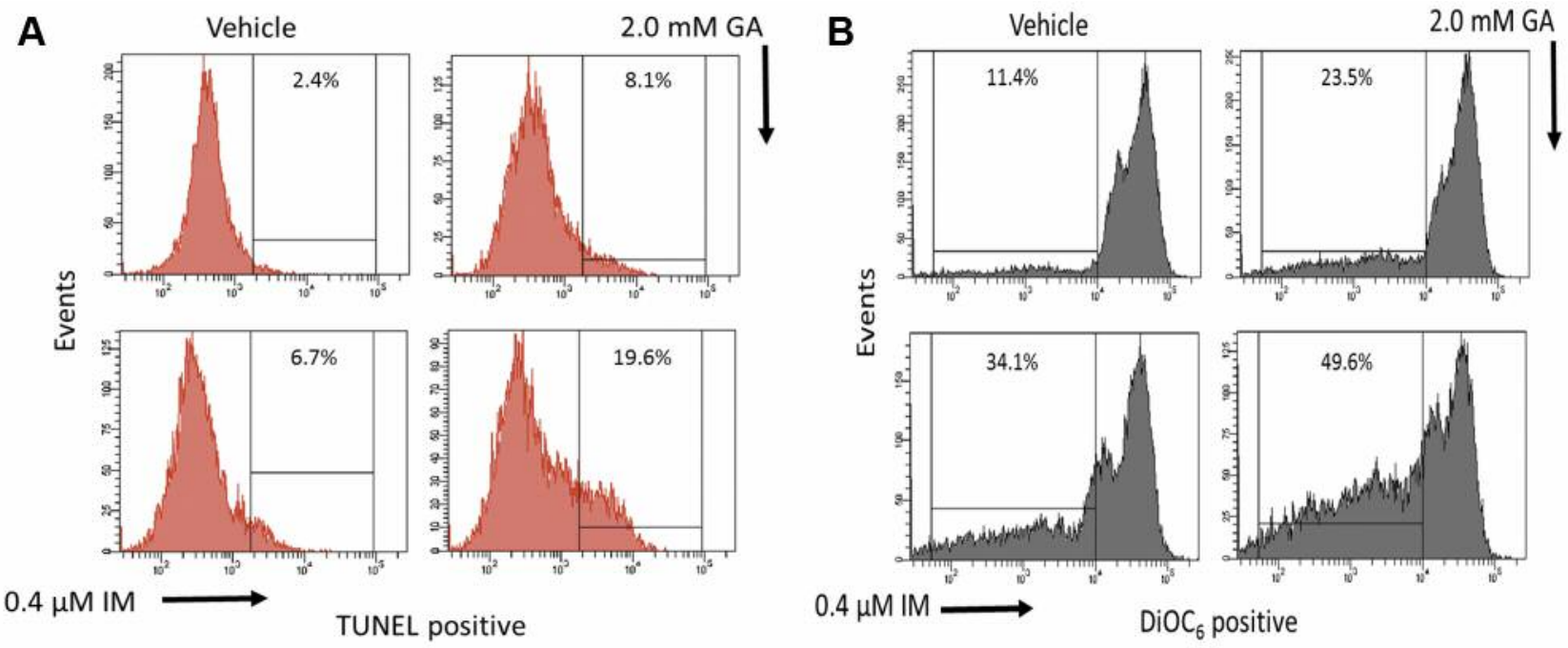

Figure 5. Co-treatment of K562 with glycrrhizic acid and imatinib enhances levels of apoptosis in vitro. K562 cultures were treated with GA (2.0 $m M)$, IM $(0.4 \mu M)$, or a combination for $48 \mathrm{~h}$. (A) The effect on the induction of apoptosis was determined by TUNEL staining. In addition, the effect on mitochondrial membrane potential was examined $(B)$. Data depicted are representative results from experiments that were conducted at least three times.
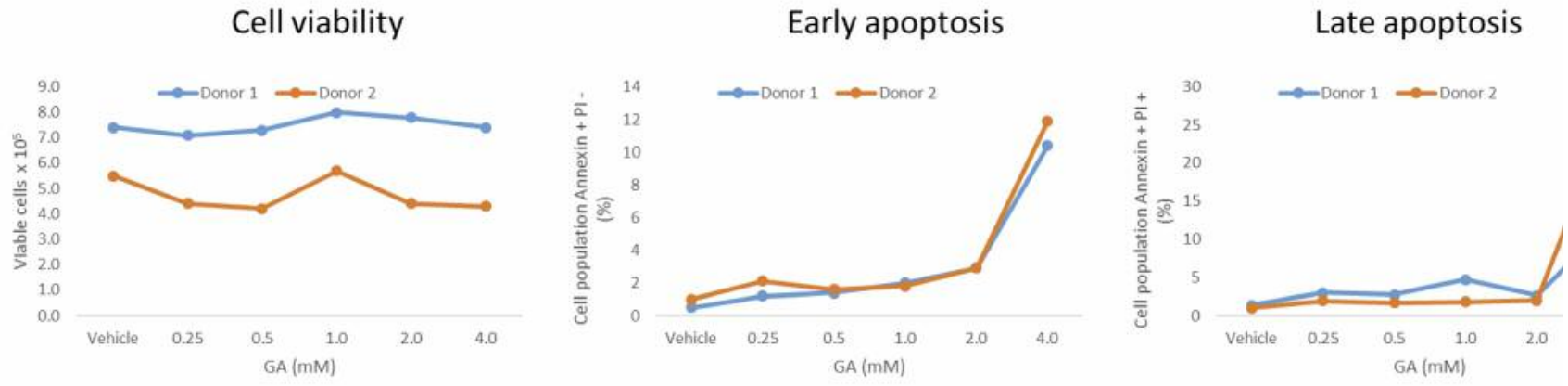

Figure 6. Low doses of glycrrhizic acid are tolerated by human PBMC's. PBMC's from healthy volunteers were isolated and treated with various concentrations of GA for $48 \mathrm{~h}$. Cell viability was assessed by trypan blue dye exclusion. PMBC cultures were also stained by Annexin V/PI for detection of early (Annexin $\mathrm{V}^{+} \mathrm{PI}^{-}$) and late apoptosis (Annexin $\mathrm{V}^{+} \mathrm{PI}^{+}$). Data depicted are from individual donors and are representative results from experiments that were conducted at least three times.

While the mechanism of GA activity against tumors is still under investigation, previous work showed that exposure to GA led to the induction of apoptosis in a number of cancer cell types $(7,15,18,24)$. Mechanistically, GA led to reduced $\mathrm{NF}-\mathrm{k} \beta$ activity in glioblastoma and in a model of colon carcinogenesis $(16,18)$. Increased caspase activity and oxidative stress were noted in leukemia and colon cancer $(16,24)$. Evidence presented in our study show that GA induces high levels of ROS generation. However, use of NAC did not lead to a significant reduction in GA-induced cell death suggesting that oxidative stress may play a role but reducing GA-induced oxidative stress in CML may not be sufficient to block GA apoptotic inducing effects. This is similar to what was reported by Hibasami et al. who demonstrated that treatment with glycyrrhetic acid, a metabolite of GA, led in increased levels of ROS and use of anti-oxidants were able to only partially protect cancer cells from glycyrrhetic acid-induced cell death (8). Additionally, similar to what was reported by Chueh et al., we found that GA causes substantial increases in DNA fragmentation and mitochondrial membrane permeabilization (24).

Single-agent therapies of malignant disease often achieve transient success but usually fail to induce durable responses. Limitations include robust survival signaling pathways, resistance developed from genomic instability, and cell heterogeneity (9). The rationale of combination 
therapy was developed to address these limitations and has been employed successfully in a wide variety of chemotherapy regimens (25). Key features of combination candidates include 1) drugs should achieve significant responses in mono-therapy, 2) administered at the highest tolerable doses, 3 ) unique mechanisms of activity to obtain synergistic responses and,4) alternative mechanisms of cellular resistance development (26). Drugs used for combination therapy ideally have independent mechanisms of toxicity so that the maximum tolerable dose for each individual agent can be given in order to achieve the highest therapeutic response. IM is a selective tyrosine kinase inhibitor that targets the BCR-ABL fusion protein present in CML and a receptor for KIT (27). It is well-tolerated in $\mathrm{CD}_{3} 4^{+}$cells in vitro up to doses of $10 \mu \mathrm{M}$ for $48 \mathrm{~h}$ far above our working dose of $0.4 \mu \mathrm{M}$ (9). Additionally, IM is relatively non-toxic in clinical applications with few sideeffects on non-malignant hematological cells (28). In the current study, co-treatment of CML with IM and GA provided a strong synergistic response, likely due to different mechanisms of action. Our study found that the maximum dose of GA tolerated by human PBMC's in vitro up to $2 \mathrm{mM}$ and so the combination with imatinib or other therapies used to treat hematological malignancies may help to maximize therapeutic effect under tolerable conditions. Therefore, combination with GA may provide more durable therapy that has less potential for the development of resistance.

The underlying intention of combination therapy is to produce a durable synergistic response that reduces the development of resistance, and does not increase cytotoxicity on normal cells. This study begins the examination of GA as a candidate for combinational therapy of CML to combat the rising resistance to current therapy. Co-treatment of CML cells with GA and IM resulted in enhanced apoptotic effects greater than either alone. The effectiveness of GA as a single agent against CML and lymphoma, its toleration by PBMCs, and boosted killing in combination with imatinib, warrants further investigations of GA as a potential combination agent not only for CML but for other hematological malignancies.

\section{References}

1 ACS: Cancer facts \& figures 2016. Atlanta: American Cancer Society, 2016.

2 ACS: Cancer treatment \& survivorship facts \& figures 20142015. Atlanta: American Cancer Society, 2014.

3 Lichtman MA: Battling the hematological malignancies: The 200 years' war. Oncologist 13(2): 126-138, 2008.

4 Gambacorti-Passerini C, Antolini L, Mahon FX, Guilhot F, Deininger M, Fava C, Nagler A, Della Casa CM, Morra E, Abruzzese E, D'Emilio A, Stagno F, le Coutre P, HurtadoMonroy R, Santini V, Martino B, Pane F, Piccin A, Giraldo P,
Assouline S, Durosinmi MA, Leeksma O, Pogliani EM, Puttini M, Jang E, Reiffers J, Valsecchi MG and Kim DW: Multicenter independent assessment of outcomes in chronic myeloid leukemia patients treated with imatinib. J Natl Cancer Inst 103(7): 553-561, 2011.

5 Alvarez-Calderon F, Gregory MA and DeGregori J: Using functional genomics to overcome therapeutic resistance in hematological malignancies. Immunol Res 55(1-3): 100-115, 2013.

6 Abe H, Ohya N, Yamamoto KF, Shibuya T, Arichi S and Odashima S: Effects of glycyrrhizin and glycyrrhetinic acid on growth and melanogenesis in cultured b16 melanoma cells. Eur J Cancer Clin Oncol 23(10): 1549-1555, 1987.

7 He SQ, Gao M, Fu YF and Zhang YN: Glycyrrhizic acid inhibits leukemia cell growth and migration via blocking akt/mtor/stat3 signaling. Int J Clin Exp Pathol 8(5): 5175-5181, 2015.

8 Hibasami $\mathrm{H}$, Iwase $\mathrm{H}$, Yoshioka $\mathrm{K}$ and Takahashi $\mathrm{H}$ : Glycyrrhetic acid (a metabolic substance and aglycon of glycyrrhizin) induces apoptosis in human hepatoma, promyelotic leukemia and stomach cancer cells. Int J Mol Med 17(2): 215219, 2006.

9 Weiss A, Berndsen RH, Ding X, Ho CM, Dyson PJ, van den Bergh H, Griffioen AW and Nowak-Sliwinska P: A streamlined search technology for identification of synergistic drug combinations. Sci Rep 5: 14508, 2015.

10 Ornstein MC, Mukherjee S and Sekeres MA: More is better: Combination therapies for myelodysplastic syndromes. Best Pract Res Clin Haematol 28(1): 22-31, 2015.

11 Chakraborty SN, Leng X, Perazzona B, Sun X, Lin YH and Arlinghaus RB: Combination of jak2 and hsp90 inhibitors: An effective therapeutic option in drug-resistant chronic myelogenous leukemia. Genes Cancer 7(5-6): 201-208, 2016.

12 Misra R and Sahoo SK: Coformulation of doxorubicin and curcumin in poly(d,1-lactide-co-glycolide) nanoparticles suppresses the development of multidrug resistance in $\mathrm{k} 562$ cells. Mol Pharm 8(3): 852-866, 2011.

13 Menegazzi M, Di Paola R, Mazzon E, Genovese T, Crisafulli C, Dal Bosco M, Zou Z, Suzuki H and Cuzzocrea S: Glycyrrhizin attenuates the development of carrageenan-induced lung injury in mice. Pharmacol Res 58(1): 22-31, 2008.

14 Chung JG, Chang HL, Lin WC, Wang HH, Yeh CC, Hung CF and $\mathrm{Li}$ YC: Inhibition of n-acetyltransferase activity and DNA2-aminofluorene adducts by glycyrrhizic acid in human colon tumour cells. Food Chem Toxicol 38(2-3): 163-172, 2000.

15 Huang RY, Chu YL, Jiang ZB, Chen XM, Zhang X and Zeng X: Glycyrrhizin suppresses lung adenocarcinoma cell growth through inhibition of thromboxane synthase. Cell Physiol Biochem 33(2): 375-388, 2014.

16 Khan R, Khan AQ, Lateef A, Rehman MU, Tahir M, Ali F, Hamiza OO and Sultana S: Glycyrrhizic acid suppresses the development of precancerous lesions via regulating the hyperproliferation, inflammation, angiogenesis and apoptosis in the colon of wistar rats. PLoS One 8(2): e56020, 2013.

17 Kobayashi M, Fujita K, Katakura T, Utsunomiya T, Pollard RB and Suzuki F: Inhibitory effect of glycyrrhizin on experimental pulmonary metastasis in mice inoculated with b16 melanoma. Anticancer Res 22(6C): 4053-4058, 2002.

18 Li S, Zhu JH, Cao LP, Sun Q, Liu HD, Li WD, Li JS and Hang $\mathrm{CH}$ : Growth inhibitory in vitro effects of glycyrrhizic acid in u251 glioblastoma cell line. Neurol Sci 35(7): 1115-1120, 2014. 
19 Ikeda K, Arase Y, Kobayashi M, Saitoh S, Someya T, Hosaka T, Sezaki H, Akuta N, Suzuki Y, Suzuki F and Kumada H: A longterm glycyrrhizin injection therapy reduces hepatocellular carcinogenesis rate in patients with interferon-resistant active chronic hepatitis c: A cohort study of 1249 patients. Dig Dis Sci 51(3): 603-609, 2006.

20 McKallip RJ, Jia W, Schlomer J, Warren JW, Nagarkatti PS and Nagarkatti M: Cannabidiol-induced apoptosis in human leukemia cells: A novel role of cannabidiol in the regulation of p22phox and nox4 expression. Mol Pharmacol 70(3): 897-908, 2006.

21 Wang ZY and Nixon DW: Licorice and cancer. Nutr Cancer 39(1): 1-11, 2001.

22 Furuya T, Yamagata S, Shimoyama Y, Fujihara M, Morishima N and Ohtsuki K: Biochemical characterization of glycyrrhizin as an effective inhibitor for hyaluronidases from bovine testis. Biol Pharm Bull 20(9): 973-977, 1997.

23 Hawthorne S and Gallagher S: Effects of glycyrrhetinic acid and liquorice extract on cell proliferation and prostate-specific antigen secretion in lncap prostate cancer cells. J Pharm Pharmacol 60(5): 661-666, 2008.
24 Chueh FS, Hsiao YT, Chang SJ, Wu PP, Yang JS, Lin JJ, Chung JG and Lai TY: Glycyrrhizic acid induces apoptosis in wehi-3 mouse leukemia cells through the caspase- and mitochondriadependent pathways. Oncol Rep 28(6): 2069-2076, 2012.

25 Pritchard JR, Lauffenburger DA and Hemann MT: Understanding resistance to combination chemotherapy. Drug Resist Updat 15(5-6): 249-257, 2012.

26 Pazdur R, Wagman LD, Camphausen KA and Hoskins WJ: Cancer management: A multidisciplinary approach. UMB Medica, 2011.

27 Yang K and Fu LW: Mechanisms of resistance to bcr-abl tkis and the therapeutic strategies: A review. Crit Rev Oncol Hematol 93(3): 277-292, 2015.

28 Peng B, Lloyd P and Schran H: Clinical pharmacokinetics of imatinib. Clin Pharmacokinet 44(9): 879-894, 2005.

Received February 8, 2017

Revised February 27, 2017

Accepted February 28, 2017 